\title{
Evaluation of The Effectiveness of China's Electricity Retail Market
}

\author{
Dezhi Li ${ }^{1, a}$ and Wenwen Zhang ${ }^{2, b}$ \\ ${ }^{1}$ China Electric Power Research institute, Beijing, 100192, China; \\ ${ }^{2}$ North China Electric Power University, Beijing, 102206, China.
}

Keywords: electricity retail market, evaluation effectiveness, grey comprehensive evaluation.

\begin{abstract}
With the gradual release of the electricity retail side in China, more and more subjects are involved in the electricity retail market. The subjects in the market achieve their needs through specific trading patterns. This paper aims to build a grey comprehensive evaluation model by constructing the evaluation index system of the operation efficiency of the electricity retail market, and to quantitatively analyze the corresponding indexes of the electricity retail market.
\end{abstract}

\section{Introduction}

Since the CPC Central Committee and the State Council issued the "Opinions on Further Deepening the Reform of the Electric Power System" and the six supporting documents, most provinces in China have actively pursued the pilot scheme of comprehensive reform or special reform pilot program. However, China lacks a set of objective and impartial evaluation system and a specialized assessment agency to support the effectiveness evaluation work of electricity retail market's regularly, scientifically and efficiently. As more and more enterprises and individuals are beginning to pay close attention to the release of the electricity market, it is necessary to develop a scientific and standardized evaluation system and model as soon as possible.

\section{The Establishment of Index System}

The reasonable setting of the index system is the scientific guarantee for the evaluation of the effectiveness of the electricity retail market. This article considers the relevant indicators from the following aspects.

(1) Market Share. For power grid enterprises, the market share should be investigated from two levels: First, the energy consumption market level. Second, the terminal energy consumption market level. The relevant indicators of electricity market share are as follows: Market share = Electricity that has been occupied / Terminal energy consumption. Direct supply market share $=$ Direct supply of electricity /Terminal energy consumption

(2) Market Concentration. Market concentration is an important feature of the market structure, used to indicate the seller or the buyer has what kind of relative size structure in an industry or market. This paper will introduce the concept of buyer concentration into the electricity retail market. Industrial organization economics usually uses the following concentration indicators:

Top-m index. Top-m index indicated with the proportion of the market's largest users that number is " $m$ " in the market share, which expressed as follows:

$$
I_{\text {Top-m }}=\sum_{i=1}^{m} S_{i}
$$

HHI index. HHI index is the most commonly used and the most representative indicators to reflect the market concentration. Its value is the sum of the square of the proportion that all users on the market electricity sales or electricity bill, defined as follows:

$$
I_{H H I}=\sum_{i=1}^{N} S_{i}^{2}
$$

(3) Typical Users 
There are two main types of typical users who have made outstanding contributions to the operating efficiency of power grid enterprises: The first, the large scale users. Scale of electricity consumption is the most important measure of the value of the enterprise to the grid. Second, the user whose power load curve and system load curve was the opposite trend.

The key index system related to the electricity market operation results as shown in the table below:

Table 1. Typical Indicators

\begin{tabular}{|c|c|c|}
\hline & Market Share: U1 & the energy consumption market level: U11 \\
\hline $\begin{array}{c}\text { Electricity Market } \\
\text { the terminal energy consumption market level: } \\
\text { Performance Indicator } \\
\text { System U }\end{array}$ & Market Concentration: U2 & $\begin{array}{c}\text { U12 } \\
\text { buyer concentration: U21 }\end{array}$ \\
\cline { 2 - 3 } & Typical Users: U3 & seller concentration: U22 \\
\hline
\end{tabular}

\section{Evaluation Model Construction}

(1) Determine the Set of Evaluation Indicators

According to the index system to establish indicators is as follows:

$\mathrm{U}=(\mathrm{U} 1, \mathrm{U} 2, \mathrm{U} 3, \mathrm{U} 4), \mathrm{U} 1=(\mathrm{U} 11, \mathrm{U} 12), \mathrm{U} 2=(\mathrm{U} 21, \mathrm{U} 22), \mathrm{U} 3=(\mathrm{U} 31, \mathrm{U} 32)$

(2) Calculate the Weight of the Indicator.

This paper uses the analytic hierarchy process to determine the weight. It calculated the weight of the primary index " $\mathrm{w}$ " and the secondary index weight " $\mathrm{w}_{\mathrm{i}}$ " according to the established index system.

(3) Determine the evaluation level standard

All the indicators are divided into excellent, good, medium, poor and bad five grades, and assigned 5, 4, 3, 2, 1 respectively. The indexes that between the two adjacent grades were assigned 4.5, 3.5, 2.5, 1.5, and the specific level of the standard determined by the experts based on experience.

(4) Construct the Grey Evaluation Weight Matrix

All evaluators denote the grey weight vector of the m-th grey type of the market to be evaluated according to the evaluation index Uij. In this study, there are five evaluation grey types, so the evaluation index Uij for the grey evaluation weight vector of each grey category rij is:

rij=(rij 1, rij2, rij3, rij4, rij5)

To sum up the gray evaluation weight vector of the gray level that comes from the second-level indicator Uij of the first-level indicator Ui, the gray evaluation weight matrix Ri are calculated.

(5) Calculate the Comprehensive Evaluation Value

The formula is as follows: $A=B * C^{T}$. Where " $A$ " is the comprehensive evaluation value, " $C$ " is the vector formed by the gray level assignment of each gray class (the first grey type takes 1 , the second grey type takes 2 , the third grey type takes 3 , the fourth grey type takes 4 , the fifth grey type takes 5$)$, and $C=(1,2,3,4,5)$. Evaluate the system comprehensively according to the grey level, and on the basis of the evaluation results, the corresponding explanation is made and the countermeasures and suggestions are put forward.

\section{Empirical Analysis}

China's electricity retail market has just started, the electricity market is in the state of not fully open, and comprehensive evaluation method of electricity market's performance is in the research stage. China has approved a series of the reform pilot in the retail side. Guangdong Province pilot is one of the earliest provinces that open up electricity retail side in China, it is representative and its research value is higher. Thus, this paper takes the electricity retail market in Guangdong Province as an example, and use grey comprehensive evaluation to assess the effectiveness of the operation of the electricity retail market. 
(1) According to the model established in this paper, the policy validity is defined as excellent, good, medium, poor, bad 5 levels, assigned 5, 4, 3, 2, 1 respectively. Assuming that five leading experts in the industry score the electricity retail market, the results are shown in Table 2.

Table 2. Expert Scoring Table

\begin{tabular}{|c|c|c|c|c|c|}
\hline Indicators & Expert1 & Expert 2 & Expert 3 & Expert 4 & Expert 5 \\
\hline U11 & 4 & 5 & 3 & 4.5 & 4 \\
\hline U12 & 4.5 & 3.5 & 4 & 3 & 4 \\
\hline U21 & 5 & 4 & 4.5 & 4 & 5 \\
\hline U22 & 3.5 & 4 & 4 & 2.5 & 4 \\
\hline U31 & 4 & 4 & 3 & 4.5 & 4 \\
\hline
\end{tabular}

(2) Assume that 10 experts in the field of electricity market score the weights of each rank of indicators, and determine the weight of the first level index $\mathrm{W}=(0.4942,0.3806,0.1252)$, the weight of the second level is: $\mathrm{W} 1=(0.6013,0.3987)$; $\mathrm{W} 2=(0.4368,0.5632)$; W3 $=(0.3891,0.6109)$.

(3) According to the implement steps of grey comprehensive evaluation, the grey evaluation weight matrix R1, R2 and R3 of the primary index of the electricity retail market are calculated.

(4) Evaluate the effectiveness of the electricity market comprehensively.

According to B1, B2, B3, Weight matrix R about total gray rating of the electricity market was got. Thus, the result of the comprehensive evaluation of the effectiveness of the market is B.

(5) Analysis of the Results of the Evaluation

By using the grey comprehensive evaluation model to analyze the corresponding indexes of the operation efficiency of the electricity retail market in China, the comprehensive evaluation value is 3.9483, and the operation effect of the electricity retail market in China is very optimistic overall. It should promote the release of the electricity market in accordance with the country's corresponding policies and documents actively.

\section{Conclusion}

In this paper, the evaluation index system of the operation efficiency of the electricity retail market is established, the grey comprehensive evaluation model is constructed, the weight of the evaluation index is determined by the importance ranking method, the grey information is evaluated by the grey whitenization weight function, and the grey clustering theory is used to determine the grey types and evaluation criteria, and then the operation of the electricity market was analyzed quantitatively. The evaluation parameters related to the effectiveness of electricity market are: market share, market concentration and typical customers of electricity market. They all promote China's electricity market liberalize and operate standardized and effectively.

\section{References}

[1] Shen Chi, Zhang Dan. Government optimization of scientific and technological resources allocation-Evaluation index system construction and policy recommendations [M]. Beijing: Peking University Press. 2013.6.

[2] Deng Ju Long. Gray theoretical basis [M]. Wuhan: Huazhong University of Science and Technology Press, 2002.

[3] Chen Meijiang. Based on the gray comprehensive evaluation method of energy-saving service company's core competitiveness evaluation system construction[J]. Zhejiang University of Technology, 2011,(9):23-28.

[4] Wu Xue. The Establishment of evaluation index system about low-carbon economic [J]. Enterprise economy, 2012 (06),12-15.

[5] Zhuang Yan. Electricity retail market's quality and its comprehensive evaluation [J]. Power System Automation, 2009 (3):3-9. 